\title{
Simulação dos efeitos dos preços de produtos e insumos na avaliação econômica de três sistemas alternativos de bovinocultura de cria
}

[Simulated effects of input and output prices on economic returns from three calf production systems]

\author{
P.H.S. Guimarães ${ }^{1}$, F.E. Madalena ${ }^{1}$, I.M. Cezar ${ }^{2}$ \\ ${ }^{1}$ Escola de Veterinária da UFMG \\ Caixa Postal 567 \\ 30123-970 - Belo Horizonte, MG \\ ${ }^{2}$ EMBRAPA - Gado de Corte - Campo Grande, MS
}

\begin{abstract}
RESUMO
Avaliou-se a sensibilidade de indicadores econômicos de três sistemas alternativos de produção de bovinos à variação de preços de insumos e produtos. Observou-se que o sistema de produção de bezerros $\mathrm{F}_{1}$ Holandês $\times$ Gir apresentou maior eficiência econômica que os sistemas de produção de bezerros Nelore e de produção de bezerros $\mathrm{F}_{1}$ Angus $\times$ Nelore, principalmente devido ao maior valor agregado de seu principal produto (novilha $\mathrm{F}_{1}$ ). $\mathrm{O}$ preço do sêmen não teve importância econômica significativa para $\mathrm{o}$ sistema de produção de bezerros $\mathrm{F}_{1}$ Holandês $\times$ Gir. Os preços da terra e da matriz Gir só tiveram importância para a avaliação econômica quando se utilizaram indicadores que levaram em conta o capital investido na atividade.
\end{abstract}

Palavras-chave: gado de corte, $\mathrm{F}_{1}$ Holandês $\times$ Gir, $\mathrm{F}_{1}$ Angus $\times$ Nelore, avaliação econômica

\begin{abstract}
By computer simulation the effect of variation in input and output prices on economic returns from three calf production systems was examined. Production of $F_{1}$ Holstein $\times$ Gir calves was more profitable than Nelore $\times$ Nelore or $F_{1}$ Angus $\times$ Nelore calf production, primarily because the market value of $F_{1}$ Holstein $\times$ Gir heifer calves was substantially higher than that of any other calf type. Variation in semen price had little impact on economic efficiency of the $F_{I}$ Holstein $\times$ Gir system. In simulations accounting for capital investment, however, prices of land and of purebred Gir cows had important influences.
\end{abstract}

Keywords: beef cattle, $F_{1}$ Holstein $\times$ Gir, $F_{1}$ Angus $\times$ Nelore, economic performance

\section{INTRODUÇÃO}

O desempenho econômico é de fundamental importância para a escolha da raça ou do cruzamento a se explorar e, embora seja dependente do desempenho zootécnico, a correlação entre ambos nem sempre é perfeita, pois o resultado de determinado cruzamento pode proporcionar maior produção que outro, porém a custo mais elevado. Assim, ao se comparar alternativas de cruzamentos, é necessário balancear de maneira apropriada todas as características zootécnicas que determinam o lucro da exploração (Madalena, 1996).

Estimar com precisão os efeitos de tomadas de decisões gerenciais e tecnológicas, considerando as possíveis interações entre componentes biológicos e econômicos associados às variações de clima e preço, nem sempre é possível.

Recebido para publicação em 30 de outubro de 2003

Recebido para publicação, após modificações, em 13 de agosto de 2004

* Autor para correspondência (corresponding author)

E-mail: fermadal@dedalus.lcc.ufmg.br 
Portanto, para minimizar riscos e erros e maximizar o objetivo principal, que é o lucro, é necessário que o responsável pela tomada de decisão disponha, a priori, de informações analíticas sobre os efeitos de possíveis decisões no sistema de produção como um todo, não só do ponto de vista biológico, mas também do econômico. Embora reconhecendo a simulação como abstração da realidade, os softwares são desenvolvidos de forma a constituir importantes ferramentas analíticas de tomadas de decisões gerenciais e tecnológicas para sistemas de produção de pecuária de corte (Cezar e Alves, 1997).

Os programas de simulação têm como objetivo servir como suporte nas tomadas de decisão e auxílio aos produtores na avaliação de decisões estratégicas numa empresa ou plano de empreendimento (Jenkins e Williams, 1999). Dessa forma, a comparação de sistemas de produção com base em simulações pode servir de base para escolha de alternativas mais eficientes e lucrativas, após as devidas validações físicas.

O objetivo deste trabalho foi avaliar, por meio de simulação ex-ante, as mudanças de preço de produtos e insumos, no desempenho econômico de sistemas de produção que têm como finalidade gerar bezerros(as) Nelore, $F_{1}$ Angus $\times$ Nelore ou $\mathrm{F}_{1}$ Holandês $\times$ Gir.

\section{MATERIAL E MÉTODOS}

Foram testados três sistemas alternativos de produção: sistema $\mathrm{N}$ (produção de bezerros Nelore), sistema AN (produção de bezerros $F_{1}$ Angus $\times$ Nelore) e sistema HG (produção de bezerros $F_{1}$ Holandês $\times$ Gir). Nas últimas duas alternativas, considerou-se também a geração das novilhas Nelore ou Gir de reposição, de forma que parte das vacas era acasalada com touros Nelore ou Gir, gerando bezerros puros, além dos mestiços.

Inicialmente foram considerados sistemas básicos (sistemas-base) para cada uma das alternativas e, em seguida, realizaram-se análises de sensibilidade, variando os preços das novilhas $\mathrm{F}_{1}(\mathrm{HG})$, das matrizes e do sêmen Gir do sistema HG e o preço da terra. Os sistemas-base foram representados por fazendas hipotéticas de cria, cujos rebanhos eram compostos de matrizes das raças Nelore (sistemas $\mathrm{N}$ e $\mathrm{AN}$ ) e Gir (sistema
HG) e estavam, inicialmente, com número estabilizado de cabeças, de acordo com a capacidade de suporte da propriedade (Guimarães, 2003). Os preços dos sistemas-base para bezerros Nelore, matriz, sêmen e terra foram, respectivamente, de: $1,63 \mathrm{R} \$ / \mathrm{kg} ; 1,06$ $\mathrm{R} \$ / \mathrm{kg} ;$ 9, $16 \mathrm{R} \$ /$ dose; 600,00 R\$/ha.

O preço da terra nos sistemas-base foi estimado levando-se em conta os preços em regiões tradicionais de pecuária de cria do estado de Minas Gerais (Anuário..., 2002). Os preços do bezerro Nelore desmamado e do bezerro mestiço leiteiro foram obtidos de uma série histórica de cinco anos (janeiro de 1997 a dezembro de 2001) e corrigidos pelo Índice Geral de Preços (IGPDI) da Fundação Getúlio Vargas (FGV) para abril de 2002.

As novilhas mestiças de leite (Holandês $\times$ Gir), em razão do seu alto potencial de produção e rusticidade, foram vendidas com ágio no preço, se comparadas às fêmeas de corte. Portanto, na situação-base, considerou-se que as novilhas mestiças tinham preço duas vezes maior que o preço do bezerro de corte (Silvestre et al., 1996; Madalena et al., 1996).

A análise foi desenvolvida a partir da simulação de sistemas produtivos de cria, assumindo que todos os bezerros (machos) foram vendidos à desmama com oito meses de idade. As bezerras desmamadas foram mantidas no rebanho até completarem um ano, quando uma parcela delas foi retida e recriada na propriedade com 0 objetivo de repor as matrizes velhas do rebanho. As novilhas excedentes foram descartadas em agosto de cada ano com aproximadamente um ano de vida (Guimarães, 2003).

Utilizou-se o programa Embrapec empregado para simulação de sistemas bioeconômicos e desenvolvido no Centro Nacional de Pesquisa de Gado de Corte - Embrapa (Cezar, 1982).

A agregação dos custos e receitas, o cálculo do fluxo de caixa e os indicadores de eficiência biológica foram executados pelo programa. As análises e as comparações entre os sistemas neste estudo foram baseadas na média de dez anos de projeção.

Para a avaliação da viabilidade econômica, foi feita uma análise baseada na metodologia proposta por Frank (1978) e utilizada por Costa 
et al. (1986) e Corrêa et al. (2000). Utilizaram-se os indicadores lucro operacional (renda líquida em dinheiro - depreciações) e resíduo para remunerar o proprietário (lucro operacional juros). Os juros foram imputados como custo de oportunidade do capital investido na atividade. Para o cálculo dos juros, utilizou-se a metodologia de valores decrescentes na qual as taxas de juros devem ser maiores para o capital circulante do que para o capital fundiário e intermediárias para o capital de exploração fixo, sendo que a taxa de juros máxima adotada foi de $6 \%$ ao ano (Guimarães, 2003). Utilizou-se ainda o retorno do capital como parâmetro de avaliação de investimentos.

Esses indicadores foram, então, avaliados sob 13 estruturas de preços, geradas por: quatro níveis de preço da novilha F1 HG (PN) em relação ao preço do bezerro, quatro níveis de preço de sêmen (PS) Gir, três níveis de preço da matriz $(\mathrm{PM})$ e quatro níveis de preço da terra (PT).

\section{RESULTADOS E DISCUSSÃO}

Nos sistemas-base o lucro operacional foi de R\$ $67.820,00$ para o sistema N, R\$ $74.055,00$ para o sistema AN e R\$126.384,00 para o sistema HG. O principal fator que levou o lucro operacional do sistema $\mathrm{HG}$ a ser, respectivamente, $86 \%$ e $70 \%$ maior que nos sistemas N e AN (Tab. 1) foi o preço da novilha $F_{1}$ Holandês $\times$ Gir. A variação dessa característica mostrou que, em cenários em que o preço da novilha do sistema $\mathrm{HG}$ foi igual a 0,8 vezes o preço do bezerro Nelore, o sistema HG apresentou lucro operacional inferior ao dos sistemas $\mathrm{N}$ e AN. Em cenários com preço da novilha $F_{1}(\mathrm{HG})$ igual a 1,2 vezes o preço do bezerro Nelore, o lucro operacional desse sistema excedeu ao dos demais.

Pôde-se observar ainda que as análises de sensibilidade para preço de matriz Gir e terra não provocaram variações no lucro operacional dos três sistemas. Isso ocorreu porque a avaliação do lucro operacional não leva em conta o capital fundiário e o de exploração fixo, os quais foram empregados nos sistemas de produção.

Quando a análise econômica foi feita por um indicador que leva em conta os juros de oportunidade do capital (Tab. 2), observou-se que a variação no preço da terra gerou uma variação muito grande na eficiência econômica dos sistemas. Em cenários em que a terra foi barata, todos os sistemas apresentaram-se eficientes economicamente. No entanto, ao se elevar o preço da terra quatro vezes mais que nos sistemas-base, apenas o sistema HG apresentouse economicamente viável.

Tabela 1. Lucro operacional (em R\$) por ano, para diferentes alternativas de produção de bezerros, avaliado sob 13 estruturas de preços, geradas por preço da novilha F1 HG $(\mathrm{PN})$, preço de sêmen Gir (PS), preço da matriz Gir (PM) e preço da terra (PT)

\begin{tabular}{ccccccc}
\hline \multicolumn{3}{c}{ Preço } & \multicolumn{3}{c}{ Sistema } \\
\hline PN & PS & PM & PT & N & AN & HG \\
\hline 0,8 & 1 & 1 & 1 & 67.820 & 74.055 & 48.625 \\
1,2 & 1 & 1 & 1 & 67.820 & 74.055 & 74.626 \\
1,6 & 1 & 1 & 1 & 67.820 & 74.055 & 100.382 \\
2 & 1 & 1 & 1 & 67.820 & 74.055 & 126.384 \\
2,4 & 1 & 1 & 1 & 67.820 & 74.055 & 152.386 \\
2 & 1,5 & 1 & 1 & 67.820 & 74.055 & 124.734 \\
2 & 2 & 1 & 1 & 67.820 & 74.055 & 123.084 \\
2 & 2,5 & 1 & 1 & 67.820 & 74.055 & 121.444 \\
2 & 1 & 2 & 1 & 67.820 & 74.055 & 126.384 \\
2 & 1 & 3 & 1 & 67.820 & 74.055 & 126.384 \\
2 & 1 & 1 & 0,5 & 67.820 & 74.055 & 126.384 \\
2 & 1 & 1 & 2 & 67.820 & 74.055 & 126.384 \\
2 & 1 & 1 & 4 & 67.820 & 74.055 & 126.384 \\
\hline
\end{tabular}

Múltiplos do preço base: bezerro Nelore $=1,63 \mathrm{RS} / \mathrm{kg}$; sêmen $=$ $9,16 \mathrm{R} \$ /$ dose; matriz $=1,06 \mathrm{R} \$ / \mathrm{kg}$; terra $=600,00 \mathrm{R} \$ / \mathrm{ha}$.

$\mathrm{N}=$ sistema de produção de bezerros Nelore; $\mathrm{AN}=$ sistema de produção de bezerros $F_{1}$ Angus $\times$ Nelore $; \mathrm{HG}=$ sistema de produção de bezerros $F_{1}$ Holandês $\times$ Gir.

O resíduo para remunerar o proprietário mostra ainda que o preço da matriz tem uma importância crescente, pois, à medida que aumenta o seu valor, aumenta também a sua participação no capital total investido na atividade. Dessa forma, quando se dobra o valor da matriz Gir do sistema base, há redução de 13,3\% nesse indicador. A partir daí, se o valor da matriz for aumentado em mais $50 \%$, provocará redução de mais $15 \%$ no resíduo para remunerar o proprietário.

É possível perceber que o indicador resíduo para remunerar o proprietário é mais sensível à variação do preço do sêmen que o lucro operacional, pois a duplicação desse preço provocou redução nesses indicadores de 3,9\% e $2,6 \%$, respectivamente. Já o retorno anual do capital investido na atividade (Tab. 3) teve sensibilidade intermediária à variação do preço do sêmen. 
Tabela 2. Resíduo para remunerar a administração $(\mathrm{R} \$)$ por ano no rebanho de cria para diferentes alternativas de produção de bezerros, avaliado sob 13 estruturas de preços, geradas por preço da novilha F1 HG $(\mathrm{PN})$, preço de sêmen Gir (PS), preço da matriz Gir (PM) e preço da terra (PT)

\begin{tabular}{ccccccc}
\hline \multicolumn{3}{c}{ Preço } & \multicolumn{3}{c}{ Sistema } \\
\hline PN & PS & PM & PT & N & AN & HG \\
\hline 0,8 & 1 & 1 & 1 & 27.313 & 33.733 & 8.910 \\
1,2 & 1 & 1 & 1 & 27.313 & 33.733 & 34.912 \\
1,6 & 1 & 1 & 1 & 27.313 & 33.733 & 60.668 \\
2 & 1 & 1 & 1 & 27.313 & 33.733 & 86.733 \\
2,4 & 1 & 1 & 1 & 27.313 & 33.733 & 112.671 \\
2 & 1,5 & 1 & 1 & 27.313 & 33.733 & 85.033 \\
2 & 2 & 1 & 1 & 27.313 & 33.733 & 83.334 \\
2 & 2,5 & 1 & 1 & 27.313 & 33.733 & 81.645 \\
2 & 1 & 2 & 1 & 27.313 & 33.733 & 75.166 \\
2 & 1 & 3 & 1 & 27.313 & 33.733 & 63.600 \\
2 & 1 & 1 & 0,5 & 36.313 & 42.733 & 95.733 \\
2 & 1 & 1 & 2 & 9.313 & 15.733 & 68.733 \\
2 & 1 & 1 & 4 & -26.686 & -20.266 & 32.733 \\
\hline
\end{tabular}

${ }^{1}$ Múltiplos do preço base: bezerro Nelore $=1,63 \mathrm{R} \$ / \mathrm{kg}$; sêmen $=$ $9,16 \mathrm{R} \$ /$ dose; matriz $=1,06 \mathrm{R} \$ / \mathrm{kg}$; terra $=600,00 \mathrm{R} \$ / \mathrm{ha}$.

$\mathrm{N}=$ sistema de produção de bezerros Nelore; $\mathrm{AN}=$ sistema de produção de bezerros $F_{1}$ Angus $\times$ Nelore; $H G=$ sistema de produção de bezerros $\mathrm{F}_{1}$ Holandês $\times$ Gir.

Tabela 3. Retorno anual do capital investido (\%) por ano no rebanho de cria para diferentes alternativas de produção de bezerros, avaliado sob 13 estruturas de preços, geradas por preço da novilha F1 HG (PN), preço de sêmen Gir (PS), preço da matriz Gir (PM) e preço da terra (PT)

\begin{tabular}{ccccccc}
\multicolumn{3}{c}{ Preço } & \multicolumn{3}{c}{ Sistema } \\
\hline PN & PS & PM & PT & N & AN & HG \\
\hline 0,8 & 1 & 1 & 1 & 5,21 & 5,81 & 3,47 \\
1,2 & 1 & 1 & 1 & 5,21 & 5,81 & 5,94 \\
1,6 & 1 & 1 & 1 & 5,21 & 5,81 & 8,38 \\
2 & 1 & 1 & 1 & 5,21 & 5,81 & 10,86 \\
2,4 & 1 & 1 & 1 & 5,21 & 5,81 & 13,31 \\
2 & 1,5 & 1 & 1 & 5,21 & 5,81 & 10,69 \\
2 & 2 & 1 & 1 & 5,21 & 5,81 & 10,53 \\
2 & 2,5 & 1 & 1 & 5,21 & 5,81 & 10,36 \\
2 & 1 & 2 & 1 & 5,21 & 5,81 & 8,84 \\
2 & 1 & 3 & 1 & 5,21 & 5,81 & 7,45 \\
2 & 1 & 1 & 0,5 & 7,24 & 8,08 & 15,18 \\
2 & 1 & 1 & 2 & 3,34 & 3,72 & 6,92 \\
2 & 1 & 1 & 4 & 1,94 & 2,16 & 4,01 \\
\hline
\end{tabular}

${ }^{1}$ Múltiplos do preço base: bezerro Nelore $=1,63 \mathrm{R} \$ / \mathrm{kg}$; sêmen $=$ $9,16 \mathrm{R} \$ /$ dose; matriz $=1,06 \mathrm{R} \$ / \mathrm{kg}$; terra $=600,00 \mathrm{R} \$ / \mathrm{ha}$.

$\mathrm{N}=$ sistema de produção de bezerros Nelore; $\mathrm{AN}=$ sistema de produção de bezerros $F_{1}$ Angus $\times$ Nelore; $H G=$ sistema de produção de bezerros $F_{1}$ Holandês $\times$ Gir.

\section{CONCLUSÕES}

Devido ao ágio pago pela novilha $\mathrm{F}_{1}$ Holandês $\times$ Gir, o sistema de produção desse tipo de animal apresentou-se mais eficiente economicamente que os sistemas de produção de bezerros Nelore e de produção de bezerros $F_{1}$ Angus $\times$ Nelore. Dentro dos limites simulados, o preço do sêmen não influenciou expressivamente os resultados econômicos do sistema de produção de bezerros $F_{1}$ Holandês $\times$ Gir. Os preços da matriz Gir e da terra só influenciaram os indicadores econômicos dos sistemas de produção quando se levou em conta o capital investido, sendo, portanto, um fator importante para as decisões de se investir ou não nessas atividades.

\section{REFERÊNCIAS BIBLIOGRÁFICAS}

ANUÁRIO DA PECUÁRIA BRASILEIRA. São Paulo: FNP, 2002.

CEZAR, I.M. Modelo bioeconômico de produção de bovino de corte. I. Descrição do modelo. Pesq. Agropec. Bras., v.17, p.941-949, 1982.

CEZAR, I.M.; ALVES, R.G.O. Embrapec: modelo bioeconômico de pecuária de corte. Campo Grande: Embrapa/CNPGC, 1997. (Manual do usuário)

CORREAA, E.S.; VIEIRA, A.; COSTA, F.P. et al. Sistema semi-intensivo de produção de carne de bovinos nelores no Centro-Oeste do Brasil. Campo Grande: Embrapa/CNPGC, 2000. (Documento, 95).

COSTA, F.P.; PACHECO, J.A.C.; CORRÊA, E.S. et al. Estimativa do custo de produção da carne bovina para a região centro-oeste: setembro de 1986. Campo Grande: Embrapa/CNPGC, 1986. (Comunicado Técnico, 30).

FRANK, R.G. Introducción al cálculo de costos agropecuarios. Buenos Aires: El Ateneo, 1978.

GUIMARÃES, P.H.S. Comparação econômica entre produção de fêmeas $F_{1}$ Holandês $\times$ Gir e alternativas de produção de gado de corte por meio de simulação. 2003. 49f. Dissertação (Mestrado em Zootecnia) - Escola de Veterinária, Universidade Federal de Minas Gerais, Belo Horizonte.

JENKINS, T.; WILLIAMS, C. Decision evaluator for the catlle industry: user's manual. Clay Center: MARC. 1999. Disponível em: <http://www.marc.usda.gov/>. Acessado em 01/08/01.

MADALENA, F.E. Pesquisa em cruzamentos de gado de leite: resultados econômicos. Cad. Téc. Esc. Vet. UFMG, n.18, p.19-27, 1996.

MADALENA, F.E.; MADUREIRA, A.P.; SILVESTRE, J.R.A. Características dos cruzamentos $\mathrm{F}_{1}$ para produção de leite em Minas Gerais. Cad. Téc. Esc. Vet. UFMG, n.18, p.41-52, 1996.

SILVESTRE, J.R.A.; MADALENA, F.E.; MADUREIRA, A.P. Fazendeiros de Minas Gerais fazem cruzamento "MeioSangue" $\mathrm{F}_{1}$ para produção de leite. Cad. Téc. Esc. Vet. $U F M G$, n.18, p.37-40, 1996. 